\title{
Superficies de titanio en implantes dentales de carga inmediata
}

\author{
C. APARICIO', A. PADRÓS', J.A. PLANELL', F.J. GIL'. \\ ${ }^{1}$ CREB. Dpto. Ciencia de los Materiales y Ingeniería Metalúrgica. \\ ETSEIB. Universidad Politécnica de Cataluña. \\ ${ }^{2}$ Instituto Padrós. Barcelona.
}

\begin{abstract}
Resumen
Se ha obtenido una capa de fosfato de calcio del mismo contenido mineral que el hueso en la superficie del implante dental de titanio, que tiene como consecuencia una muy rápida y eficaz osteointegración. Esta capa se ha obtenido mediante un método biomimético por vía termoquímica. La capa se une al substrato por enlace químico. Este hecho evita todos los inconvenientes de los métodos de recubrimiento convencionales (plasma spray, ablación láser...) cuya unión es por interacción mecánica, lo cual provocaba que hubiese espacio vacio entre el substrato y la capa facilitando la colonización bacteriana (periimplantitis) y un desprendimiento y degradación acelerados de la misma. Con la formación de la capa por el método termoquímico, sobre la superficie rugosa del implante, el tiempo de convalecencia se reduce significativamente ya que colocamos una estructura en la superficie del implante que las células reconocen, facilitando el crecimiento óseo alrededor del implante dental. A esta capacidad de formar capas cerámicas de fosfato de calcio en la superficie del biomaterial se la conoce como bioactividad y, anteriormente, era una habilidad que sólo tenían algunas cerámicas y vidrios. El desarrollo y obtención de estos implantes de titanio con una rugosidad optimizada y bioactivos combina una magnífica fijación mecánica con la rapidez del crecimiento del hueso, con lo que se consigue un implante con mejor osteointegración tanto a corto como a largo plazo.
\end{abstract}

\section{Introducción}

Los implantes dentales se fabrican mayoritariamente de titanio comercialmente puro y de Ti-6Al-4V debido a que son metales con buenas propiedades mecánicas, buena resistencia a la corrosión, excelente biocompatibilidad y, por lo tanto, una gran capacidad de osteointegración, es decir, el hueso es capaz de crecer a su lado. Esta capacidad de osteointegración provoca que el implante dental no se mueva y no se afloje con el tiempo en servicio. Sin embargo, el aflojamiento es la causa principal del fracaso de los implantes dentales. La deficiente colonización del hueso al lado del implante conlleva una movilidad que, con los ciclos mecánicos masticatorios, conduce al

\author{
Correspondencia: \\ C. Aparicio \\ CREB. Dpto. Ciencia de los Materiales y Ingeniería \\ Metalúrgica. \\ ETSEIB. Universidad Politécnica de Cataluña. \\ Diagonal 647 - 08028, Barcelona (España)
}

fracaso del implante. La posterior extracción del mismo es cruenta y además, en muchos casos, el paciente queda con una deficiencia de hueso; situación en la que la colocación de un nuevo implante dental va a ser muy complicada.

Un problema clínico adicional son los largos periodos de convalecencia del paciente. Estos son debidos a que, antes de poder poner la superestructura dental y, sobre todo, de permitir que el implante soporte los esfuerzos mecánicos masticatorios con normalidad, se debe esperar a que el hueso colonice la superficie del implante dental. Durante este periodo, que oscila entre cuatro y seis meses, el paciente se ve afectado en sus hábitos alimenticios, en su habla y en su imagen.

Atendiendo a todo ello, este proyecto se ha desarrollado con el objetivo de mejorar las condiciones de osteointegración de los implantes dentales a corto plazo. Esto se ha conseguido aportando valores de innovación en la tecnología de los tratamientos y procesos superficiales que se llevan a cabo en el implante dental durante su fabricación, para que éste interaccione con el tejido óseo circundante de forma más rápida y eficiente. 


\section{Materiales y Métodos}

\section{Obtención de implantes Dentales Bioactivos}

La apatita es un fosfato de calcio con la misma composición química que la fase mineral del hueso, lo que implica que es completamente aceptada en el cuerpo como una sustancia propia. No habrá reacciones inflamatorias importantes, si se incorpora en el cuerpo como un implante. No sólo eso, sino que además tiene la ventaja de que una vez implantada, el tejido óseo crece sobre ella con enlace químico directo, comportándose como lo que se denomina un material bioactivo (1-4).

La innovación en este estudio reside en que se desea realizar el depósito de la apatita químicamente en titanio rugoso tratado con arenado, es decir, se quiere conseguir una mayor rapidez de osteointegración (reducir el período en el que el paciente no debe realizar cargas bucales) gracias a la bioactividad de la apatita depositada y a la vez conseguir una buena fijación a largo plazo, ya que cuando se forme hueso nuevo, éste colonizará la rugosidad del titanio producida por el método de areando y se conseguirá además de una fijación química una fijación mecánica. Es decir, se busca combinar los dos procedimientos (superficie bioactiva y rugosidad) para conseguir una mayor fijación a corto plazo (bioactividad) y a largo plazo (rugosidad) (5-7)

Este método consiste, de manera resumida, en conseguir que la superficie de titanio pase a ser bioactiva, gracias a la formación de un gel denso y amorfo de titanato de sodio, inducida por un tratamiento químico y térmico. En este momento, si el titanio se implanta in vivo, ya es capaz de formar enlace químico directo con el hueso ordenado. Si se deposita una capa de apatita in vitro antes de la implantación, el tejido humano no ha de realizar las primeras etapas de enlace con el implante.

Para conseguir el depósito de apatita in vitro sobre el titanio ya tratado química y térmicamente, es decir, con el gel en superficie, se sumerge el implante dentro de una solución que contiene una concentración iónica muy cercana a la del plasma sanguíneo humano (Tabla I). Debido a esta similitud se le llama Simulated Body Fluid (SBF) (8-10).

\section{Tratamiento termoquímico biomimético}

En el método empleado las placas de titanio son tratadas con una solución de $\mathrm{NaOH}$ a una cierta temperatura durante 24 horas para formar un gel de un titanato de sodio. Luego se limpian en agua destilada y se secan a $40^{\circ} \mathrm{C}$ durante 24 horas. Después sigue un tratamiento térmico para que el gel pase a ser más denso y así mejorar la adhesión entre éste y el titanio. La adhesión aumenta con la temperatura, pero para temperaturas superiores a $600^{\circ} \mathrm{C}$ el gel cristaliza en un alto porcentaje, lo que retarda el posterior proceso de deposición de apatita sobre la superficie. El mecanismo de formación de apatita depende de la migración de los iones de sodio, procedentes del hidrogel de titanato de sodio formado en la superficie del Ti c.p. después de su tratamiento con $\mathrm{NaOH}$ (9-11).

Estas moléculas cargadas negativamente se combinan con iones de sodio en la solución acuosa lo que resulta en la formación de una capa de hidrogel de titanato de sodio. Durante el tratamiento térmico, este hidrogel se deshidrata y se densifica para formar una capa de titanato de sodio estable y amorfo. Cuando se sumerge en SBF se hidrata otra vez y se transforma en un hidrogel de $\mathrm{TiO}_{2}$ por una liberación de iones de sodio de la capa de titanato de sodio a la solución de SBF. Si el hidrogel se cristaliza, la liberación de estos iones va a tardar más tiempo, lo que retarda todo el proceso de nucleación de la apatita (12-15).

El paso de liberación de sodio está acompañado por un cambio de iones con $\mathrm{H}_{3} \mathrm{O}^{+}$del $\mathrm{SBF}$ que resulta en un aumento del $\mathrm{pH}$ de la solución. El aumento del $\mathrm{pH}$ produce un aumento del producto de actividad iónica de la apatita, lo que resulta en la rápida deposición de la misma sobre la superficie de titanio según la reacción de equilibrio siguiente:

$$
10 \mathrm{Ca}^{2+}+6 \mathrm{PO}_{4}^{3-}+2 \mathrm{OH}^{-} \Leftrightarrow \mathrm{Ca}_{10}\left(\mathrm{PO}_{4}\right)_{6}(\mathrm{OH})_{2}
$$

Con esta reacción el ratio de supersaturación con respecto a la apatita en el SBF, que ya antes de sumergir los substratos es fuertemente supersaturado, aumenta aún más. Éste es un proceso que hace que la titania hidratada induzca la nucleación de apatita sobre la superficie del titanio. Gracias a la supersaturación, muchos núcleos de apatita se forman como bolas pequeñas por toda la superficie del Ti c.p. Una vez la nucleación ha empezado, los núcleos crecen espontáneamente, consumiendo los iones de calcio y de fosfato que contiene el SBF.

La caracterización de las capas formadas se realizó mediante la observación en un Microscopio Electrónico de Barrido Ambiental (ESEM) y la caracterización analítica de los productos se realizó mediante estudios de Difracción de Rayos X rasantes. Estos estudios se realizaron a diferentes tiempos para ir observando la cinética de reacción. Se determinó también la rugosidad antes y a diferentes tiempos del tratamiento para ver su 
influencia y su posible variación con la deposición de las capas (16-19).

Las observaciones iniciales en el ESEM mostraron que, después de los tratamientos químicos y térmicos, se creó una estructura esquelética, porosa, en la superficie de las placas tratadas con $\mathrm{NaOH}$ (Figura 1). Esta estructura se encuentra también sobre las partículas de $\mathrm{Al}_{2} \mathrm{O}_{3}$ y de $\mathrm{SiC}$, que habían quedado encastadas en la superficie del Ti c.p. después del tratamiento de arenado (Figura 2). Esta estructura será el substrato para el crecimiento de la capa apatítica (20).

Al tercer día de inmersión en SBF se pudieron observar en ciertos puntos de las placas rugosas

\begin{tabular}{|c|c|c|}
\hline Ion & SBF & Plasma humano \\
\hline $\mathrm{Na}^{+}$ & 142.0 & 142.0 \\
\hline $\mathrm{K}^{+}$ & 5.0 & 5.0 \\
\hline $\mathrm{Mg}^{2+}$ & 1.5 & 1.5 \\
\hline $\mathrm{Ca}^{2+}$ & 2.5 & 2.5 \\
\hline $\mathrm{Cl}^{-}$ & 147.8 & 103.0 \\
\hline $\mathrm{HCO}_{3}^{-}$ & 4.2 & 27.0 \\
\hline $\mathrm{HPO}_{4}^{2-}$ & 1.0 & 1.0 \\
\hline $\mathrm{SO}_{4}^{2-}$ & 0.5 & 0.5 \\
\hline
\end{tabular}

Tabla 1. Concentración iónica $(\mathrm{mM})$ del SBF y del plasma sanguíneo humano
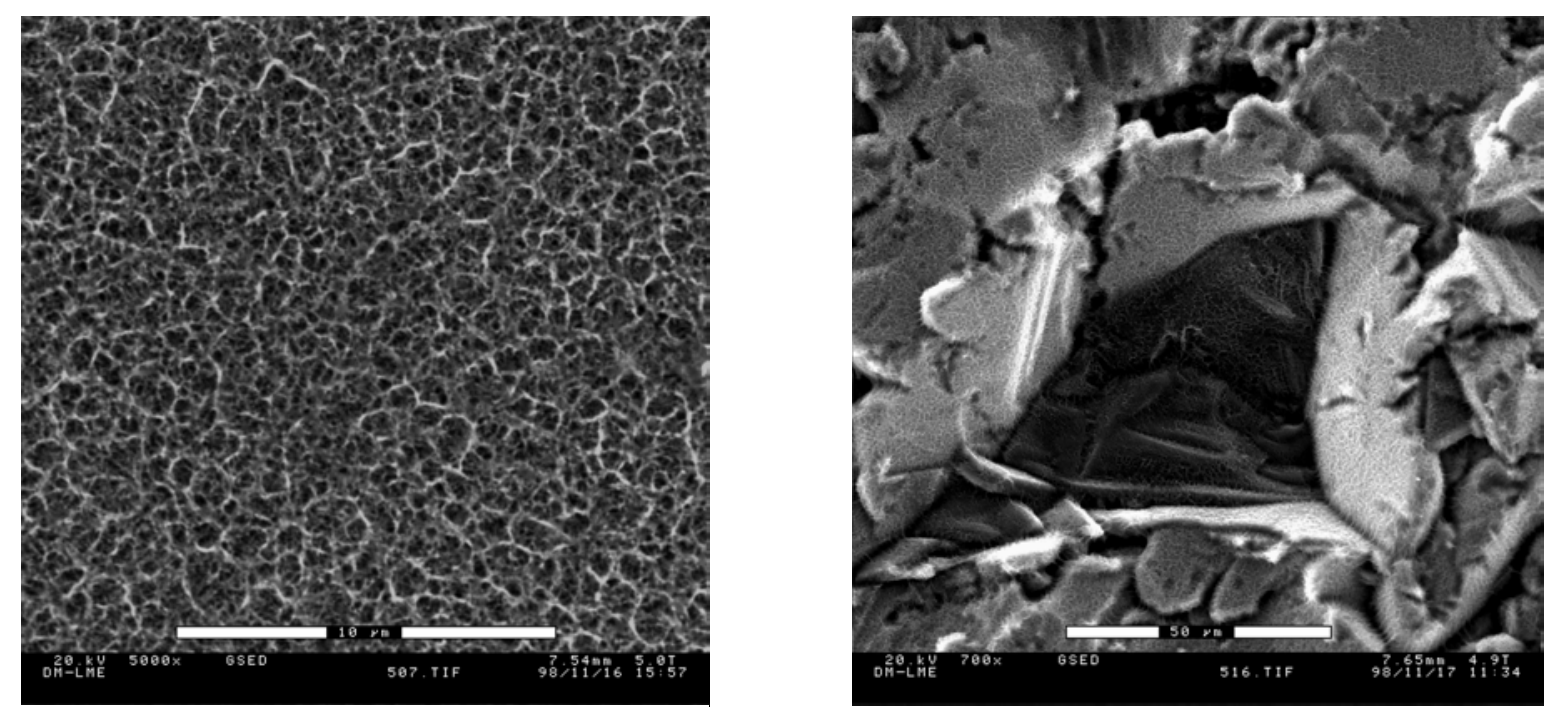

Figura 1 (izquierda). Estructura de titanato de sodio sobre una placa lisa

Figura 2 (derecha). Estructura de titanato de sodio sobre una partícula de SiC 
tratadas con partículas de $\mathrm{Al}_{2} \mathrm{O}_{3}$, pequeñas agrupaciones de cristales muy finos (del orden del nanómetro). (Figura 3). Se observó que había más cristales sobre las partículas de $\mathrm{Al}_{2} \mathrm{O}_{3}$ que sobre la superficie del Ti c.p., lo que puede ser una evidencia de que la presencia de $\mathrm{Al}_{2} \mathrm{O}_{3}$ influye, en la cinética de la nucleación de apatita acelerándola. Al cuarto día de inmersión en SBF, las placas tratadas con partículas de $\mathrm{Al}_{2} \mathrm{O}_{3}$, pasivada y/o lavada, y atacada con $\mathrm{NaOH}$ estaban recubiertas por una capa homogénea de apatita (como se comprobó con la difracción). En cambio, en las placas tratadas con partículas de $\mathrm{SiC}$ y en las placas lisas todavía no se observaba más que la estructura esquelética de titanato de sodio.

Después de cinco días de tratamiento en SBF había capas homogéneas recubriendo toda la superficie de las placas lisas. Estas capas eran del mismo tipo que los encontradas en las placas tratadas con partículas de $\mathrm{Al}_{2} \mathrm{O}_{3}$, pasivadas y lavadas, antes descritas (20-23).

Al final del proceso de deposición de apatita, es decir después de 11 días de inmersión en el SBF no se observaron cambios del aspecto de la capa homogénea de apatita depositada para las muestras lisas y los granallados con $\mathrm{Al}_{2} \mathrm{O}_{3}$ (Figura 4). Los difractogramas de rayos $\mathrm{X}$ rasantes de las placas de $\mathrm{Al}_{2} \mathrm{O}_{3}$ al final del tratamiento confirmaron que la apatita crece con el tiempo del tratamiento con SBF. Los máximos de apatita aumentan el número de cuentas entre los cuatro y los once días. Esto significa principalmente que la capa homogénea aumenta, habiendo más cristales de apatita en profundidad desde la superficie, haciéndose más significativa la presencia de esta fase en el difractograma de Rayos X rasantes.

Por último, en las placas tratadas con partículas de SiC después de 11 días en el SBF, se observaron depósitos semejantes a los núcleos de apatita, por medio de ESEM. Sin embargo, los depósitos no tenían exactamente la misma morfología que la apatita. No eran tan redondeados como las pequeñas esferas de apatita $\mathrm{y}$, por lo tanto, se suponía que eran depósitos de otra sustancia. Esto fue confirmado por medio de la difracción de rayos $\mathrm{X}$.

Las observaciones iniciales en el ESEM mostraron que, después de los tratamientos químicos y térmicos, se creó una estructura esquelética, porosa, en la superficie de las placas tratadas con $\mathrm{NaOH}$ (Figura 1). Esta estructura se encuentra también sobre las partículas de $\mathrm{Al}_{2} \mathrm{O}_{3}$ y de $\mathrm{SiC}$, que habían quedado encastadas en la superficie del Ti c.p. después del tratamiento de arenado (Figura 2). Esta estructura será el substrato para el crecimiento de la capa apatítica (20).

Al tercer día de inmersión en SBF se pudieron observar en ciertos puntos de las placas rugosas tratadas con partículas de $\mathrm{Al}_{2} \mathrm{O}_{3}$, pequeñas agrupaciones de cristales muy finos (del orden del nanómetro). (Figura 3). Se observó que había más cristales sobre las partículas de $\mathrm{Al}_{2} \mathrm{O}_{3}$ que sobre la superficie del Ti c.p., lo que puede ser una evidencia de que la presencia de $\mathrm{Al}_{2} \mathrm{O}_{3}$ influye, en la cinética de la nucleación de apatita acelerándola. Al cuarto día de inmersión en SBF, las placas tratadas con partículas de $\mathrm{Al}_{2} \mathrm{O}_{3}$, pasivada y/o lavada, y atacada con $\mathrm{NaOH}$ estaban recubiertas por una capa homogénea de apatita (como se comprobó con la difracción). En cambio, en las placas tratadas con partículas de $\mathrm{SiC}$ y en las placas lisas todavía no se observaba más que la estructura esquelética de titanato de sodio.

Después de cinco días de tratamiento en SBF había capas homogéneas recubriendo toda la superficie de las placas lisas. Estas capas eran del mismo tipo que los encontradas en las placas tratadas con partículas de $\mathrm{Al}_{2} \mathrm{O}_{3}$, pasivadas y lavadas, antes descritas (20-23).

Al final del proceso de deposición de apatita, es decir después de 11 días de inmersión en el SBF no se observaron cambios del aspecto de la capa homogénea de apatita depositada para las muestras lisas y los granallados con $\mathrm{Al}_{2} \mathrm{O}_{3}$ (Figura 4). Los difractogramas de rayos $\mathrm{X}$ rasantes de las placas de $\mathrm{Al}_{2} \mathrm{O}_{3}$ al final del tratamiento confirmaron que la apatita crece con el tiempo del tratamiento con SBF. Los máximos de apatita aumentan el número de cuentas entre los cuatro y los once días. Esto significa principalmente que la capa homogénea aumenta, habiendo más cristales de apatita en profundidad desde la superficie, haciéndose más significativa la presencia de esta fase en el difractograma de Rayos X rasantes.

Por último, en las placas tratadas con partículas de SiC después de 11 días en el SBF, se observaron depósitos semejantes a los núcleos de apatita, por medio de ESEM. Sin embargo, los depósitos no tenían exactamente la misma morfología que la apatita. No eran tan redondeados como las pequeñas esferas de apatita y, por lo tanto, se suponía que eran depósitos de otra sustancia. Esto fue confirmado por medio de la difracción de rayos $X$.

Uno de los resultados más importantes de este estudio es que la apatita crece con la misma cinética sobre toda la placa. Es decir, la capa no va a alcanzar un aspecto liso con el tiempo, sino que la rugo- 
sidad va a mantenerse durante la deposición (Tabla II). Este hecho ya se podía esperar al observar las micrografías de la capa de apatita sobre las muestras tratadas con $\mathrm{Al}_{2} \mathrm{O}_{3}$.

Así pues, se ha demostrado que se puede utilizar el método de Kokubo et al. para depositar químicamente capas homogéneas de apatita sobre una superficie de Ti c.p. lisa, así como una rugosa tratada con partículas de óxido de aluminio, lo cual permite controlar la rugosidad final obtenida ya que la deposición de la capa no altera los valores de $\mathrm{R}_{\mathrm{a}}$ para las placas rugosas. El hecho de que la rugosidad se mantenga, deja abierta la posibilidad de que la fijación de los implantes dentales se pueda mejorar a largo plazo; por un lado, porque las propiedades mecánicas de la pieza (vida a fatiga, resistencia a la corrosión, etc.) mejoran con el tratamiento de arenado y el implante se fija mecánicamente cuando el hueso crece dentro de los hoyuelos en su superficie $(20,24)$.

Pero la apatita mejora, sobre todo, la fijación a corto plazo. La experiencia muestra que el hueso reconoce esta capa homogénea como una sustancia propia y no se producen las reacciones inflamatorias, obteniéndose porcentajes de implante en contacto directo con hueso vivo y ordenado a tiempos muy cortos. Debido a este hecho, el proceso de remodelación del hueso empieza antes, y los tiempos de convalecencia de los pacientes disminuyen con los beneficios sociales y económicos consiguientes. Otro aspecto ventajoso que presenta la capa homogénea de apatita es que aísla las partículas que han quedado incrustadas en la superficie después del tratamiento de arenado. De esta manera, se supone que se pueden evitar en gran medida los posibles efectos contaminantes de las partículas y que la liberación de iones disminuirá y/o desaparecerá por completo.

\section{Evaluación de los implantes bioactivos in vivo}

El estudio ha tenido como objetivo evaluar la osteointegración a corto y medio plazo de implantes rugosos y/o bioactivos, ya que si esta evaluación es positiva, indica que los implantes son susceptibles de ser implantados en humanos, como último paso.

Se ha llevado a cabo una implantación in vivo (mandíbula de cerdo) de histomorfometría ósea como medida de la osteointegración de los implantes. Los implantes estudiados se han tratado mediante un arenado i/o un tratamiento termoquímico para obtener superficies bioactivas.

Los implantes (mecanizado) utilizados en los ensayos eran de titanio comercialmente puro de grado III. Sobre estos implantes se llevaron a cabo distintos tratamientos. Así, habían implantes implantes arenados con partículas de óxido de aluminio de $600 \mu \mathrm{m}$ de tamaño (arenado), con el mimso anterior pero sólo hasta el cuello del implante (noemal), con un tratamiento con ácido fluorhídrico (ácido) y con el tratamiento de arenado y el método termoquímico para conferirles bioactividad

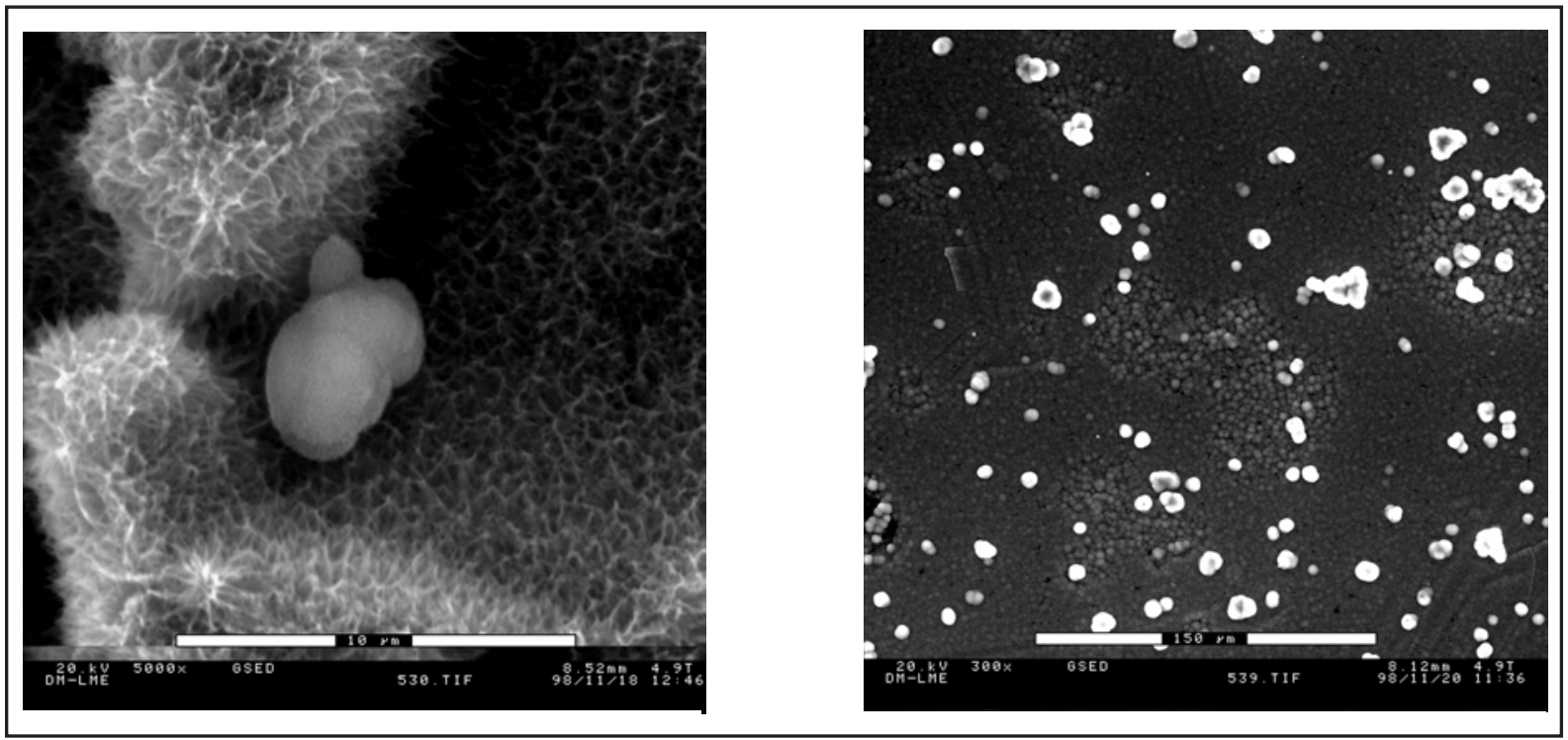

Figura 3 (izquierda). Agrupaciones de cristales en la placa rugosa tratada con $\mathrm{Al}_{2} \mathrm{O}_{3}$ y lavada, después de 3 días en SBF

Figura 4 (derecha). Capa homogénea de apatita en placa lisa después de 5 días en SBF 
(bioactivo). En este caso interesa comparar los mecanizado, los arenado y los normal.

Se implantaron 100 implantes en los huesos maxilares de 5 microcerdos, 20 en cada cerdo. Cada cerdo tenía 4 implantes de cada uno de los tipos descritos anteriormente. Los cerdos se sacrificaron después de 2, 4, 6, 8 y 10 semanas, y de cada implante se obtuvo una muestra para su análisis histomorfométrico.

La histomorfometría consiste en medir la cantidad de hueso en contacto directo con el implante. Esta medición se lleva a cabo tras la preparación de muestras histológicas del implante y los tejidos circundantes de cada tipo de implante después de cada tipo de implantación. Cuanto mayor sea la cantidad de hueso en contacto directo con el implante mayor será la fijación y, por lo tanto, mejor la osteointegración.
En cuanto a los fracasos en la implantación, cabe destacar que los implantes bioactivos no fracasaron en ningún caso, lo cual no fue conseguido por ninguno de los otros tipos de implantes. Esto ya es un resultado muy significativa en cuanto a la bonanza de los implantes rugosos y bioactivos.

El resultado más importante de este estudio fue que los implantes bioactivos, a diferencia del resto de los implantes estudiados, consiguieron valores de $\%$ de hueso en contacto con el implante al cabo de 4 semanas que ya eran significativamente (estadísticamente) superiores a los obtenidos a las 2 semanas (Figura 5). Esto indica que los implantes bioactivos se osteointegran más rápidamente que los otros tipos. Por lo tanto, corrobora que el objetivo perseguido con todas las investigaciónes se ha conseguido. Sobre todo si se tiene en cuenta que a más largo plazo, es decir, 10 semanas, los implantes

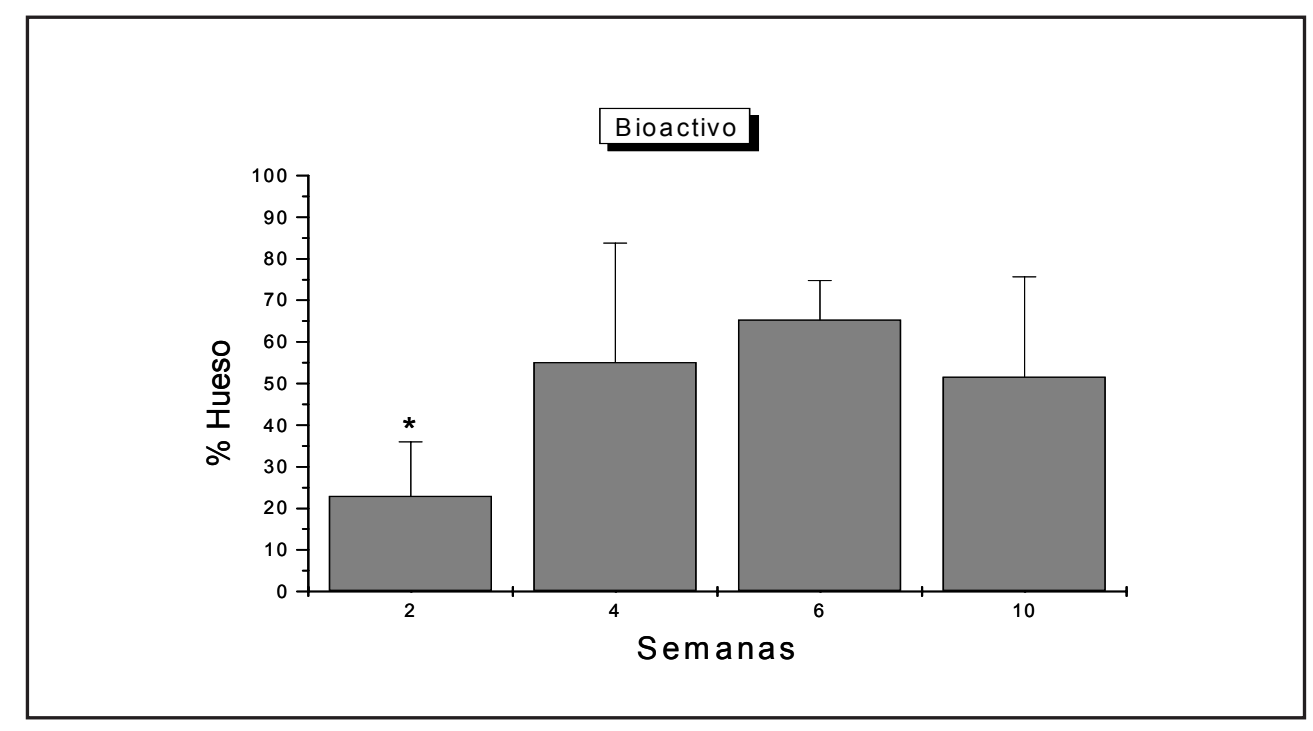

Figura 5. \% de hueso en contacto directo con el implante para los implantes Bioactivo según el tiempo de implantación. (*) Diferencias estadísticamente significativas

\begin{tabular}{|c|c|c|c|c|}
\hline \multirow[t]{2}{*}{ Placas } & \multicolumn{2}{|c|}{ Antes tratamiento } & \multicolumn{2}{|c|}{ Método termoquímico } \\
\hline & $\mathrm{R}_{\mathrm{a}}$ medio & D. est. & $\mathrm{R}_{\mathrm{a}}$ medio & D. Est \\
\hline Lisa & 0,07 & 0,02 & 0,38 & 0,16 \\
\hline $\mathrm{Al}_{2} \mathrm{O}_{3}$ & 3,93 & 0,21 & 3,88 & 0,21 \\
\hline $\mathrm{SiC}$ & 3,66 & 0,27 & 3,84 & 0,21 \\
\hline
\end{tabular}

Tabla 2. Valores de Rugosidad $\left(\mathrm{R}_{\mathrm{a}}\right.$ en $\left.\mu \mathrm{m}\right)$ para las muestras estudiadas. D. est $=$ Desviación estándar 
bioactivos mantienen altos valores de ostointegración (Figura 5).

Las Figura 6 muestra un detalle de un corte histomorfométrico de un implante bioactivo al cabo de 4 semanas y en la Figura 7 se puede ver el mismo tipo de detalle, pero para un implante mecanizado al cabo de 10 semanas. La mayor osteointegración del implante rugoso y bioactivo a tiempos mucho más cortos se hace evidente al observar el hueso crecido en ambos casos. Comparando con los implantes arenados al cabo de 10 semanas (Figura 8), se aprecia que las diferencias con el implante bioactivo al cabo de 4 semanas tampoco es significativa, lo que indica, de nuevo, la gran velocidad de osteointegración de los implantes bioactivos.

\section{Conclusiones}

Como conclusión general, pues, se puede destacar que los resultados nos indican que los implantes rugosos con superficies bioactivas demuestran un osteointegración más rápida que el resto de los implantes.

\section{Agradecimientos}

Los autores agradecen a la empresa de implantes dentales Klockner, y en especial a la Sra. Mercedes Roldán Chesa, motor y soporte científico y económico del presente trabajo. Asimismo, los autores desean agradecer a la CICYT la concesión del proyecto de investigación que ha sufragado en parte este estudio.

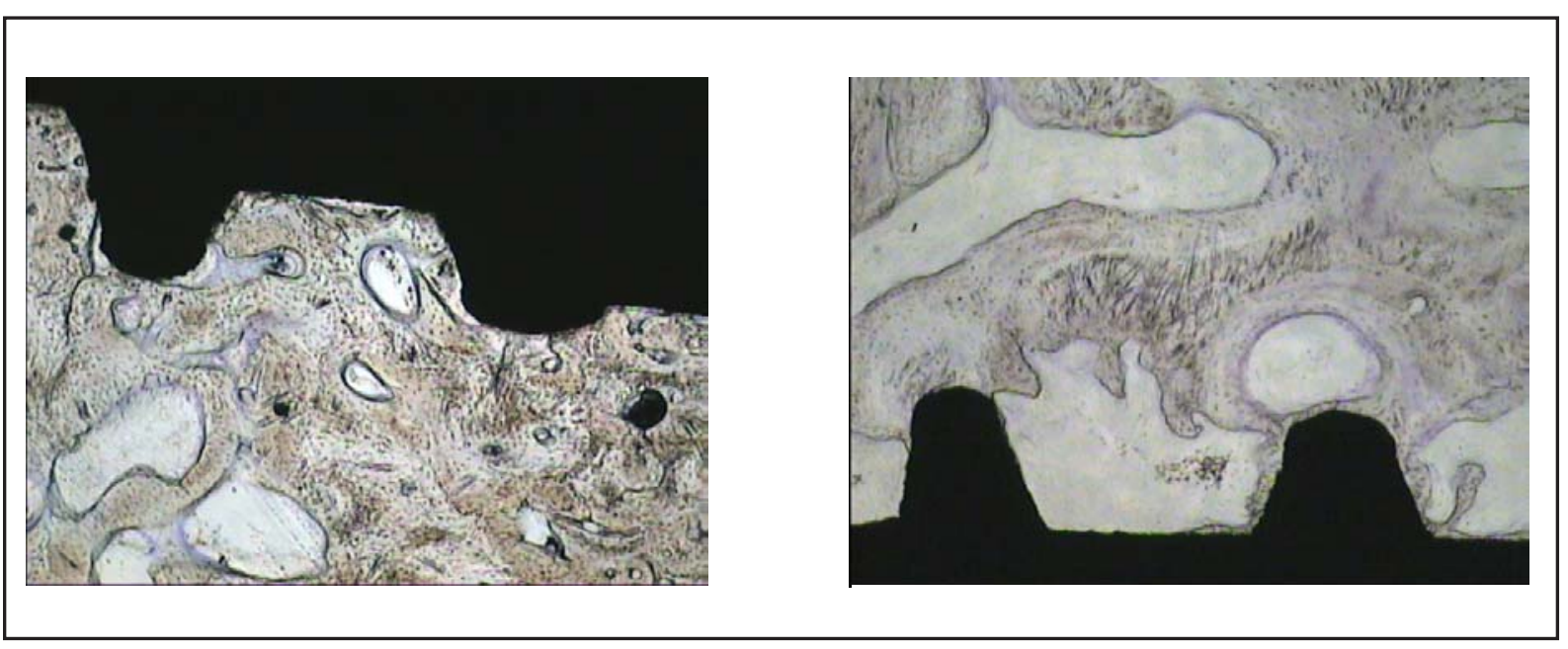

Figura 6 (izquierda). Histología de implante rugoso y bioactivo después de 4 semanas implantado

Figura 7 (derecha). Histología de implante mecanizado al cabo de 10 semanas de implantación

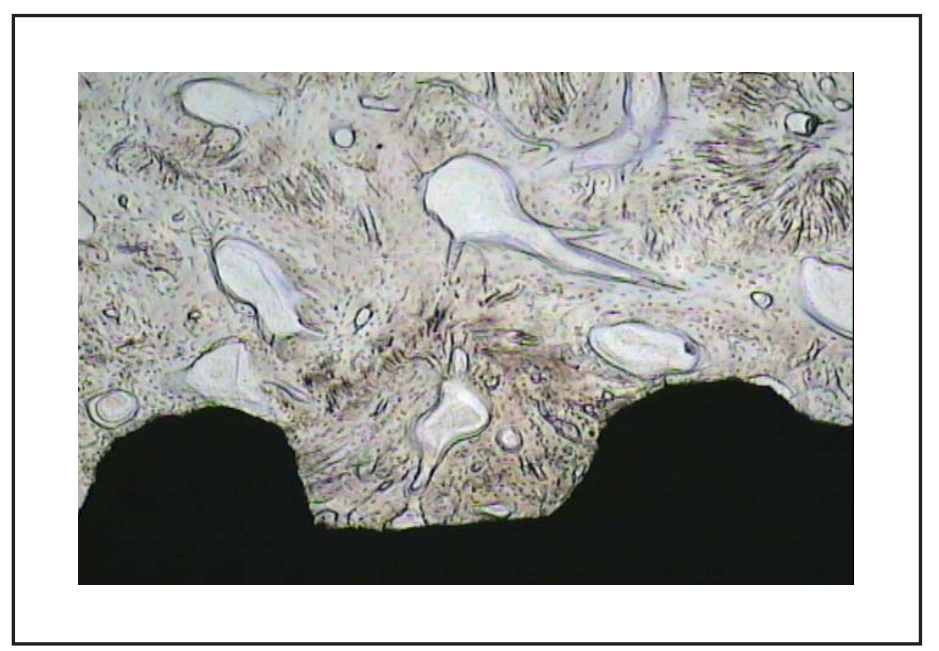

Figura 8. Histología de implante arenado al cabo de 10 semanas de implnatación 


\section{Bibliografía}

1. Kim HM, Kokubo T, Fujibayashi S, et al. Bioactive macroporous titanium surface layer on titanium substrate. J Biomed Mater Res. 2000; 52: 553-7.

2. S.L. Carlsson, T.R. Rostlund, B. Albrektsson, T. Albrektsson and P.-I. Brånemark. «Osseointegration of titanium implant» Acta Orthop. Scand., 57, 285-289 (1986).

3. L. Sennerby, P. Thomsen and L.E Ericson. «Ultrastructure of the bone-titanium interface in rabbits» Mater. Med., 3, 262-271 (1992).

4. K. Takatsuka, T. Yamamuro, T Nakamura and T. Kokubo. «Bone-bonding behaviour of titanium alloy evaluated mechanically with detaching failure load» J. Biomed. Mater. Res., 29, 157-164 (1995).

5. L.L. Hench and Ö. Anderson. «Bioactive glass coating» in An Introduction to Bioceramics. L.L. Hench and J. Wilson (eds) World Science Singapore, pp. 239-260. (1993).

6. K. Takatsuka, T. Yamamuro, T. Kitsugi, T. Nakamura, T. Shibuya and Goto. «Anew bioactive glass-ceramic as a coating material on titanium alloy» J. Appl. Biomater., 4, pp. 317-329 (1993).

7. K. De Groot, R.G.T. Geesink, C.P.A.T. Klein and P. Serekian. «Plasma sprayed coatings of hydroxyapatite» J. Biomed. Mater. Res., 21, 1375 1387 (1987).

8. C.Y. Yang, R.M. Lin, B.C. Wang, T.M. Lee, E. Chang, Y.S. Hang and P.Q. Chen. «In vitro and in vivo mechanical evaluations of plasma-sprayed hydroxyapatite coatings on titanium implants: The effect of coating characteristics» J. Biomed. Mater. Res., 37, 335-345 (1997).

9. T. Kokubo, F. Mijyaji and H.-M. Kim. «Spontaneous formation of bonelike apatite layer on chemically treated titanium metals» J. Am. Ceram. Soc., 79, 1127-1129(1996).

10. W.Q. Yan, T. Nakamura, M.Kobayashi, H.-M. Kim, F. Miyaji and T. Kokubo. «Bonding of chemically treated titanium implants to bone» J. Biomed. Mater. Res., 37, 267-275 (1997).

11. T. Kokubo. «Novel bioactive materials». Anales de Química Int. Ed. pp. S49-55 (1997).

12. H. Takadama, H.M. Kim, F. Miyaji. T. Kokubo and T. Nakamura. «How does alkali-treated titanium induce apatite formation» in Bioceramics Vol 11. Edited R.Z. LeGeros and J.P. LeGeros. pp. 663- 666.

13. T. Hurlen and W.Wihelmsen. "Passive behaviour of titanium» Electrochim. Acta 31, (1986) 1139-1146.

14. K.E.Healy and P.Ducheyne. «Passive dissolution kinetics of titanium in vitro». J.Mater.Sci.Mater.Med. 4, (1993) 117-126.

15. K.E.Healy and P.Ducheyne. «The mechanism of passive dissolution kinetics of titanium in a model physiological environemnt». J.Biomed. Mater. Res. 36(1992)319-338.

16. L.D.Arsov, C.Kormman, and W.Plieth. «In situ Raman spectra of anodically formed titanium dioxide layer in solution of $\mathrm{H}_{2} \mathrm{SO}_{4}, \mathrm{KOH}$, and $\mathrm{HNO}_{3} »$ Electrochem. Soc. 138, (1991) 2964-2970.

17. H.M.Kim, F.Miyaji, T.Kokubo and T. Nakamura. «Preparation of bioactive $\mathrm{Ti}$ and its alloys via simple surface treatment». J. of Biomed. Mater. Res. 32 (1996) 409-417.

18. J. Gamble. «Chemical Anatomy Physiology and Pathology of Extracellular Fluid». $6^{\text {th }}$ edition, Harvard University Press, Cambridge, MA (1967).

19. W. Neuman and M.Neuman. The Chemical Dynamics of Bone Mineral. University of Chicago Press, Chicago IL, (1958).

20. L. Cleries. "In vitro studies of calcium phosphate coatings obtained by laser ablation». PhD Thesis, University of Barcelona, Spain, 1999.

21. H. Hero, H. Wie, R.B. Jorgensen, I.E. Ruyter. «Hydroxyapatite coating on titanium produced by isostatic pressing». J. Biomed. Mater. Res. 28, 344348, 1994.

22. K. De Groot, C.P. Klein, J.G. Wolke, J.M. BlieckHogervorst. «Plasma sprayed coatings of calcium phosphate». In: CRC Handbook of Bioactive Ceramics. Vol. II. Calcium phosphate and hydroxyapatite ceramics». Ed. T. Yamamuro, L. Hench, J. Wilson. CRC Press, Inc. 133-142, 1990.

23. A.S. Posner, N.C. Blumenthal, F. Betts. In: Proceedings of the $2^{\text {nd }}$ International Conference on Phosphorous Compounds. Ed. Eon, C. Imphos, Paris, p. 25, 1980.

24. E. Park, S.R. Condrate, D.T. Hoelzer, G.S. Fischman. «Interfacial characterisation of plasmaspray coated calcium phosphate on Ti6Al4V». J. Mater. Sci. Med. 9, 643-649, 1998. 